\title{
Fault Detection and Diagnosis of Induction Machines based on Wavelet and Probabilistic Neural Network
}

\author{
Pu Shi \\ Engineering Department \\ Glyndwr University \\ Wrexham, UK
}

\author{
Zheng Chen \\ Engineering Department \\ Glyndwr University \\ Wrexham, UK
}

\author{
Yuriy Vagapov \\ Engineering Department \\ Glyndwr University \\ Wrexham, UK
}

\begin{abstract}
In this paper, a prototype wavelet and probabilistic based neural network classifier for recognizing rotor bar defects is implemented and tested under various transient signals. The wavelet transform (WT) technique is integrated with the neural network model to extract rotor fault features. Firstly, the multiresolution analysis technique of WT and the particle swarm optimization (PSO) theorem are employed to extract the features of the distorted signal. Then, the probabilistic neural network (PNN) classifies these extracted features to identify the rotor defects type. The proposed approach can reduce a great quantity of the distorted signal features without losing its original property. Moreover, less memory space and computing time are required. Various experimental cases tested results show that the hybrid classifier can detect and classify broken rotor bar faults efficiently.
\end{abstract}

\section{Keywords}

Induction machine, WT, PSO, PNN, Broken rotor bar.

\section{INTRODUCTION}

Induction machines have playing an essential role in manufacturing plants. Although induction motors are designed to be robust and rigidly, motor failures are expect to happen sooner or later due to their harsh work conditions. These failures may yield unexpected interruption during industrial processing and cause serious consequences in costs, product quality, and safety issues.

On-line monitoring and early detection of abnormalities in the motors will help avoid unexpected failures and reduce downtime frequency. Motivated by this industrial demand, several diagnosis approaches for the identification and discrimination of the enumerated faults have been proposed. Nandi and Toliyat studied temperature measurements, infrared recognition, radio frequency emissions, noise monitoring or chemical analysis [1]. Kliman, et al. monitored the motor axial flux by using search coils to detection broken rotor bars [2]. Cameron and Thomson proposed to use vibration and current monitoring for detecting airgap eccentricity in large induction motors [3]. Schoen, et al. introduced an unsupervised, on-line system for induction motor fault detection using motor stator current spectrum signature analysis [4]. Cruz, et al. and Nejjari, et al. presented park's vector current approach to monitor and diagnosis of induction motors separately [5] [6]. Salles, et al. proposed artificial intelligence based techniques to monitor induction motor load [7]

Among different detection approaches proposed in the literature, those based on stator current monitoring are advantageous due to its non-invasive characteristic. From all these approaches proposed in the publications, artificial intelligence based approach have been in the central research field in recent years.

Neural networks have become a popular approach in induction machine faults diagnosis due to their fault tolerance and their capability for self-organization. Among these artificial networks, the multilayer perception (MLP), and the back-propagation (BP) training algorithm are probably the most frequently used neural networks in practical applications [8]. However, both these two networks have some inherent defects, such as low learning speed, easy trapped in local minima, and difficult to choose proper parameters of network.

To overcome these drawbacks, a hybrid approach that integrates wavelet with neural network and employed a novel training algorithm is proposed in this paper. The wavelet neural network (WNN) has proved its ability as a novel universal approach for functional approximation, which shows surprising effectiveness in solving the conventional problem of poor convergence as well as increase convergence speed [9] [10] [11] [12].

This paper proposes an on-line current monitoring system to perform the diagnosis task in a supervisory system. In section 2 , the architectures of wavelet neural networks and the training algorithm are introduced. Overview of probabilistic neural network (PNN) is described in section 3. In section 4, experimental test-rig design is demonstrated. In section 5, design of hybrid WNN and PNN are simulated and applied to intelligent fault diagnosis. Finally, conclusions are presented in section 6 .

\section{WA VELET NEURAL NETWORK (WNN)}

The wavelet neural network (WNN) employed in this research is designed as a three-layer structure with an input layer, wavelet layer (hidden layer) and output layer. Figure 1 illustrated the topological configuration of the WNN, where $w_{j k}$ denotes the weights connecting the input layer and the hidden layer, $u_{i j}$ denotes the weights connecting the hidden layer and the output layer. In this WNN models, the hidden neurons have wavelet activation functions of different resolutions, the output neurons have sigmoid activation functions. A similar architecture can be used for generalpurpose function approximation and system identification.

The activation functions of the wavelet nodes in the wavelet layer are derived from a mother wavelet $\Psi(x)$, suppose $\Psi(x) \in L^{2}(R)$, which represents the collection of all measurable functions in the real space, satisfies the admissibility condition [13] 


$$
\int_{-\infty}^{+\infty} \frac{|\Psi(\omega)|^{2}}{\omega} d \omega<\infty
$$

Where $\Psi(\omega)$ indicates the Fourier transform of $\Psi(x)$. The output of the wavelet neural network $\boldsymbol{Y}$ is represented by the following equation:

$$
y_{i}=\sigma\left(x_{n}\right)=\sigma\left(\sum_{j=0}^{M} u_{i j} \psi_{a, b}\left(\sum_{k=0}^{L} w_{j k} x_{k}(t)\right)\right)
$$

$(i=1,2, \ldots, N)$

Where $\sigma\left(x_{n}\right)=1 /\left(1+e^{-x_{n}}\right), y_{i}$ denotes the $j$ th component of the output vector; ${ }^{x_{k}}$ denotes the $k$ th component of the input vector; $u_{i j}$ denotes the connection weight between the output unit $i$ and the hidden unit $j ; w_{j k}$ denotes the weight between the hidden unit $\mathrm{j}$ and input unit $k ; a_{j}, b_{j}$ denote dilation coefficient and translation coefficient of wavelons in hidden layer respectively; $L, M, N$ denote the sum of input, hidden and output nodes respectively.

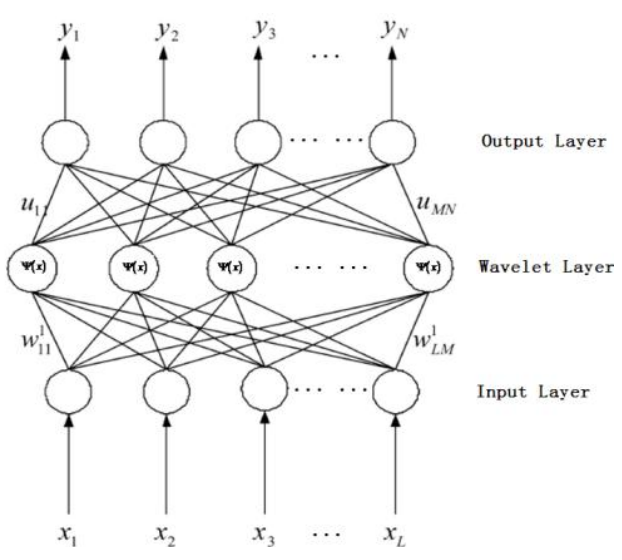

Fig 1. The WNN topology Structure

The network is trained with a hybrid algorithm integrating particle swarm optimization (PSO) with gradient descent algorithm in batch way [14]. As a result, the network model in this paper is constructed by using PSO as training algorithm and Morlet mother wavelet basic function as node activation function [14].

\subsection{Particle Swarm Optimization (PSO)}

Particle swarm optimization approach (PSO) first introduced by Kennedy and Eberhart is one of the modern heuristic algorithms and has been adapted in various engineering applications. PSO is an evolutionary computation technique motivated by the simulation of social behaviour and has attracting attentions in both research field and industrial applications due to its evolutionary algorithms (EAs) [15] [16] [17]. It is characterized as a simple heuristic of well balanced mechanism with flexibility stochastic search technique to enhance and adapt to both global and local exploration abilities [17].

PSO conducts searches using a population of particles which correspond to individuals in evolutionary algorithm. The PSO system initially randomly generates a population of solutions. Each potential solution represented by a particle and has a position represented by a position vector $x_{i d}$. A swarm of particles moves through the problem space in a random velocity. Each particle keeps tracking its own best position, which is associated with the best fitness it has achieved so far, named pbest. Furthermore, a number of pbest for the respective particles achieved so far and the greatest fitness particle is called the global best (gbest) of the swarm. In addition to this global version, another version of PSO keeps track of the best position among all the topological neighbours of a particle.

During the iteration procedure, the velocity of particle $i$ is updated according to the following equation [14]:

$$
v_{i d}^{k+1}=\chi\left(w v_{i d}^{k}+c_{1} \phi_{1} \times\left(p_{i d}-x_{i d}^{k}\right)+c_{2} \phi_{2} \times\left(p_{g d}-x_{i d}^{k}\right)\right)_{(3)}
$$

Where, $k$ is the current iteration number. The velocity of the rate of the position change for particle $i$ is represented as $V_{i}=$ $\left(v_{i 1}, v_{i 2}, \ldots, v_{i D}\right), v_{i}^{k}$ is the current velocity of particle $i$ at iteration $k, v_{i}^{k+1}$ is the modified velocity of particle $i$ at iteration $k+1$.The $i$ th particle is treated as a point in a $D$ dimensional space and represented as $X_{i}=\left(x_{i 1}, x_{i 2}, \ldots, x_{i D}\right), x_{i}^{k}$ is the current position of particle $i$ at iteration $k$. The best previous position of the $i$ th particle (pbest ${ }_{i}$ ) that gives the best fitness value is represented as $P_{i}=\left(p_{i 1}, p_{i 2}, \ldots, p_{i D}\right), p_{i d}$ is the pbest ofparticle $i$. The best particle among all the particles in the population is represented by $P_{g}=\left(p_{g 1}, p_{g 2}, \ldots, p_{g D}\right), p_{g d}$ is the gbest of the group. $\chi$ and $w$ are the weight function for velocity of particle $i, c 1$ and $c 2$ are the weight coefficientsfor each term, $\phi_{1}$ and $\phi_{2}$ are uniformly distributed random number between 0 and 1

Using the above equation, a certain velocity that gradually gets close to pbest and gbest can be calculated. The current position (searching point in the solution space) can be modified by the following equation [14]:

$$
x_{i d}^{k+1}=x_{i d}^{k}+v_{i d}^{k+1}
$$

Velocities of particles on each dimension are clamped by a maximum velocity $V_{\max }$. If the sum of accelerations would cause the velocity on that dimension to exceed $V_{\max }$, which is a parameter specified by the user, then the velocity on that dimension is limited to $\mathrm{V}_{\max } . \mathrm{V}_{\max }$ influences $\mathrm{PSO}$ performance sensitively. A larger $V_{\max }$ facilitates global exploration, while a smaller $\mathrm{V}_{\max }$ encourages local exploitation [18].

The constants $c 1$ and $c 2$ represent the weighting of the stochastic acceleration terms that pull each particle toward pbest and gbest positions. Low values allow particles to roam far from target regions before being tugged back. On the other hand, high values result in abrupt movement toward, or past, the target regions. Hence, the acceleration constants $\mathrm{c} 1$ and $\mathrm{c} 2$ were often set to be 2 according to past experience.

The inertia weight $w$ is introduced in to improve PSO performance. The model using (5) is called "inertia weights approach (IWA)" Suitable selection of inertia weight $w$ provides a balance between global and local exploration and exploitation, and on average results in less iterations required to find a sufficiently optimal solution. As originally developed, the inertia weight $w$ is set according to the following equation [14]: 


$$
w=w_{\max }-\frac{w_{\text {max }}-w_{\text {min }}}{\text { iter }_{\max }} \times k
$$

where $w_{\max }$ is the initial weight, $w_{\min }$ is the final weight, iter $_{\max }$ maximum iteration number.

\section{PROBABILISTIC NEURAL NETWORK (PNN)}

The probabilistic neural network (PNN) was first introduced by Specht in 1990 and is a form of neural network designed for classification [19]. A PNN is implemented by using the probabilistic models, such as Bayesian classifiers and has distinct features from those of other network in the learning processes. A PNN is guaranteed to converge to a Bayesian classifier provided enough training data because it has no required to learning processes, initial weights of the network and no relationship between learning processes and recalling processes.

The learning speed of the PNN model is very fast, making it suitable for fault diagnosis and signal classification problems in real time. PNN can be implemented as a neural network, which is shown in Figure 2 [20]. The network architecture of a PNN is determined by the number of compounds and descriptors in the training set. There are 4 layers in a PNN. The input layer provides input values to all neurons in the pattern layer and has as many neurons as the number of descriptors in the training set. The number of pattern neurons is determined by the total number of compounds in the training set. Each pattern neuron computes a distance measure between the input compound and the training compound represented by that neuron and then subjects the distance measure to the Parzen's nonparameteric estimator. The summation layer has a neuron for each data class and the neurons sum all the pattern neurons' output corresponding to members of that summation neuron's data class to obtain the estimated probability density function for that data class. The single neuron in the output layer then determines the final data class of the input compound by comparing all the probability density functions from the summation neurons and choosing the data class with the highest value for the probability density function.

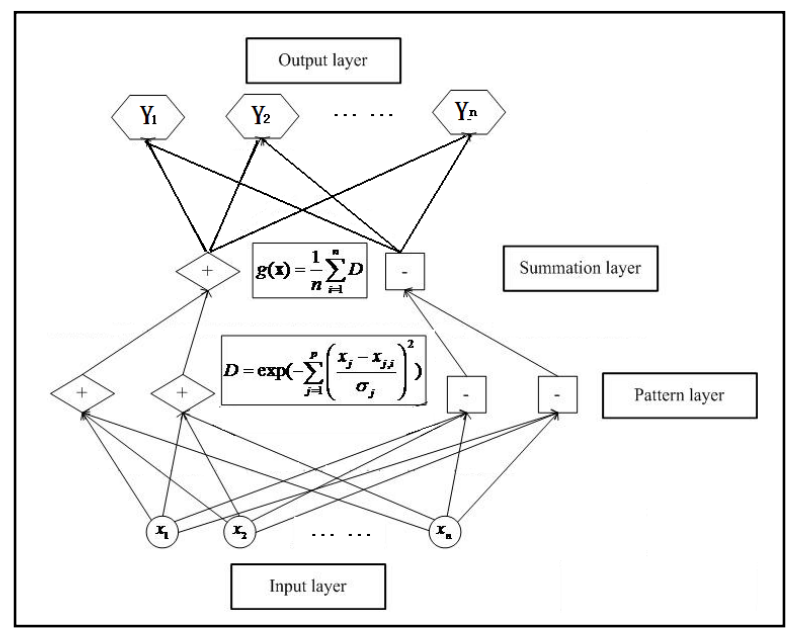

Fig 2. Architecture of a PNN

In the signal classification application, the training examples are classified according to their distribution values of probabilistic density function (PDF), which is the basis principle of the PNN. A simple PDF is defined as follows [19]:

$$
f_{k}(X)=\frac{1}{N_{k}} \sum_{j=1}^{N_{k}} \exp \left(-\frac{\left\|X-X_{k j}\right\|}{2 \sigma^{2}}\right)
$$

A form of neural network designed for classification through the use of the Bayes' optimal decision rule:

$$
h_{i} c_{i} f_{i}(x)>h_{j} c_{j} f_{j}(x)
$$

where $h_{i}$ and $h_{j}$ are the prior probabilities, $c_{i}$ and $c_{j}$ are the costs of misclassification and $f_{i}(x)$ and $f_{j}(x)$ are the probability density function for data class $i$ and $j$ respectively. A given compound with vector $\mathrm{x}$ is classified into data class $i$ if the product of all the three terms is greater for data class $i$ than for any other data class $j$ not equal to $i$. In most applications, the prior probabilities and costs of misclassifications are treated as being equal. The probability density function for each data class for a univariate case can be estimated by the Parzen's nonparametric estimator [21]:

$$
g(x)=\frac{1}{n \sigma} \sum_{i=1}^{n} W\left(\frac{x-x_{i}}{\sigma}\right)
$$

where $n$ is the sample size, sigma is a scaling parameter which defines the width of the bell curve that surrounds each compound, $W(d)$ is a weight function which has its largest value at $d=0$ and $\left(x-x_{i}\right)$ is the distance between a given compound and a compound in the training set. The Parzen's nonparametric estimator is expanded by Cacoullos for the multivariate case [22].

$$
g\left(x_{1,}, \ldots, x_{p}\right)=\frac{1}{n \sigma_{1} \ldots \sigma_{p}} \sum_{i=1}^{n} W\left(\frac{x_{1}-x_{1, i}}{\sigma_{1}}, \cdots, \frac{x_{p}-x_{p, i}}{\sigma_{p}}\right)
$$

The Gaussian function is frequently used as the weight function because it is well behaved, easily calculated and satisfies the conditions required by Parzen's estimator. Thus the probability density function for the multivariate case becomes

$$
g(x)=\frac{1}{n} \sum_{i=1}^{n} \exp \left(-\sum_{j=1}^{p}\left(\frac{x_{j}-x_{j, i}}{\sigma_{j}}\right)^{2}\right)
$$

To simplify the equation, a single sigma that is common to all the descriptors (single-sigma model) can be used instead of an individual sigma for each descriptor (multi-sigma model). Single-sigma models could be computed faster and can produce reasonable models when all the descriptors are of approximately equal importance. However, multi-sigma models are more general than single-sigma model and are useful when descriptors are of different nature and importance [20].

\section{EXPERIMENTAL TEST-RIG DESIGN}

In order to test and verify the performance of the condition classification system, a whole experimental set based on the proposedsystem were configured. The experiment was carried out under the self-designed test rig which is composed of three phase induction machines, current transducer, A/D converter, and computer as shown in Figure 3.

The specifications of the 3 phase induction machines used in this experiment are listed in Table 1. Among these tests, one isnormal (healthy), which was set as a benchmark conditioncompared with other faulty motors with one, two and three broken rotor bars. A current Hall Effect sensor was 
placed in one of the line current cables. The stator current was sampled with a $1 \mathrm{kHz}$, rate and interfaced to a PC by an ADC11 acquisition board.

Table 1. Specifications of an Induction Machine

\begin{tabular}{|c|c|}
\hline Specification & Value \\
\hline Rated Power & $3.7 \mathrm{~kW}$ \\
\hline Horse Power & $5 \mathrm{HP}$ \\
\hline Input Voltages & $220 / 380 \mathrm{~V}$ \\
\hline Input Currents & $13.8 / 8.0 \mathrm{~A}$ \\
\hline Pole pairs & 2 \\
\hline Frequency & $50 \mathrm{~Hz}$ \\
\hline Speed & $3000 \mathrm{rpm}$ \\
\hline
\end{tabular}

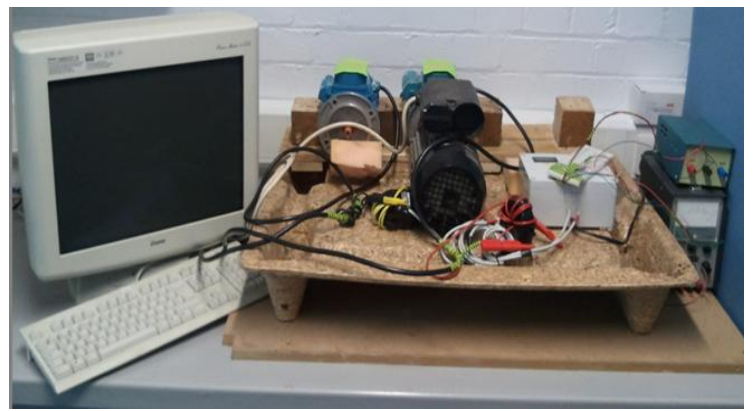

Fig 3. View of the experimental setup and broken rotor bars

\section{FEATURE EXTRACTION AND FAULT CLASSIFICATION}

Different fault feature extraction techniques have been proposed in the literature, of which stator current based approach is the most employed non-invasive method for monitoring of motor faults, especially broken rotor bars. Other techniques such as speed, torque, vibration, acoustic and air gap flux need additional transducers and extra equipments to be fitted with the motor for measuring which may interrupt the operation. Moreover, extra problem arises as the sensor sensitivity changes due to environmental effect which makes the measurement unreliable.

A series of experiments have been conducted to verify the validity and effectiveness of the fault detection scheme proposed in this paper. The stator current signals are employed to detect broken bar faults and to classify the conditions since the signals describe the dynamic characteristics of the induction motor.

\subsection{Stator Currents Features Analysis and Extraction}

Stator current signature is a cost effective alternative monitoring technique because of its easy availability. Flourier transform is the most used approach to analyse stator current at steady state based on detection of the side band harmonics close to supply frequency, or its other harmonics according to $(1 \pm 2 s) f$, where, $f$ is the supply frequency and $s$ is the slip, $k=1,2,3, \ldots$

This phenomenon is related to amplitude modulation (AM) of stator current induced by rotor structure modification due to broken rotor bars as stated in [23]. However, the presence of stator current AM cannot be detected without accurate time or frequency analysis. Tradition Flourier transform method of analysis of the steady state current involves some important drawbacks when encounter transient signal. In order to analysis transient signal, wavelet transform is employed because of it has a very appealing feature of uniform resolution at each scale.

In this paper, only main stator current frequency band is considered according to $(1 \pm 2 s) f$ broken bars detection criteria. Db 10 wavelet transform is used to filter out the rest these unexpected signals.

Figure 4(a). illustrates motor stator current performances for healthy case under different load level, (b). shows the boundary appearance corresponds to Figure 4(a). Stator current amplitudes increasing are clear noticed as 0, 40\%, $80 \%$ and $100 \%$ load level shown from top to bottom respectively. Figure 5. presents stator currents performances for two broken bars under same operation conditions as Figure 4. Figure 6. presents stator currents for $0,1,2$ and 3 broken bars under $60 \%$ load level. There are no obvious stator current amplitudes changes under same load level comparing Figure 4. and Figure 5. However, there are riffles exist in the broken bars stator currents due to speed fluctuations. These broken bars caused riffles are not obvious at low load level and small broken bars number.

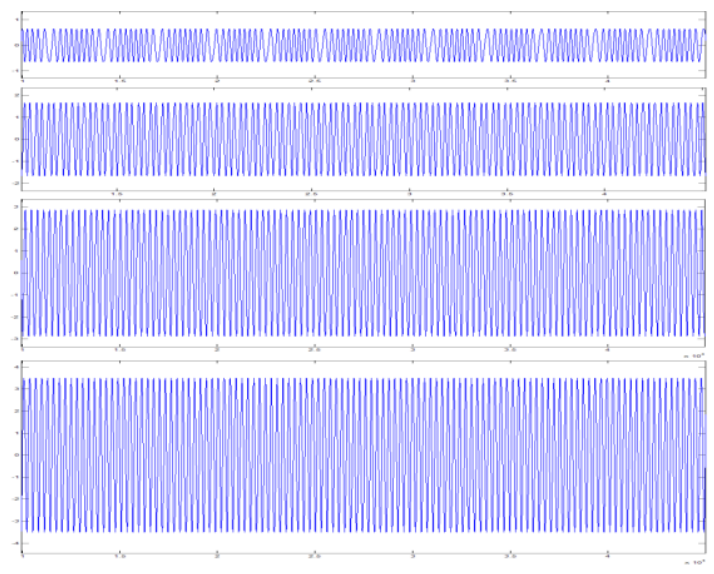

a. $\quad$ Stator currents
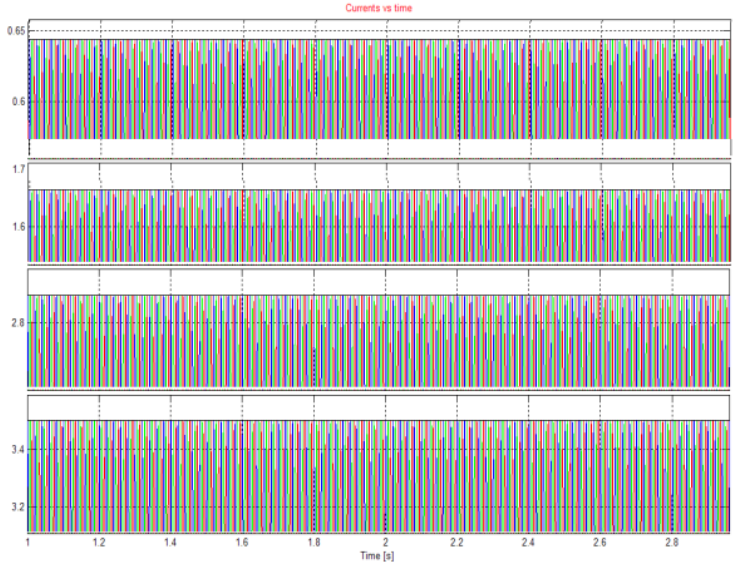

b. Stator current boundary appearance

Fig.4. Stator currents for healthy motor under $0,40 \%$, $80 \%$ and $100 \%$ load. 

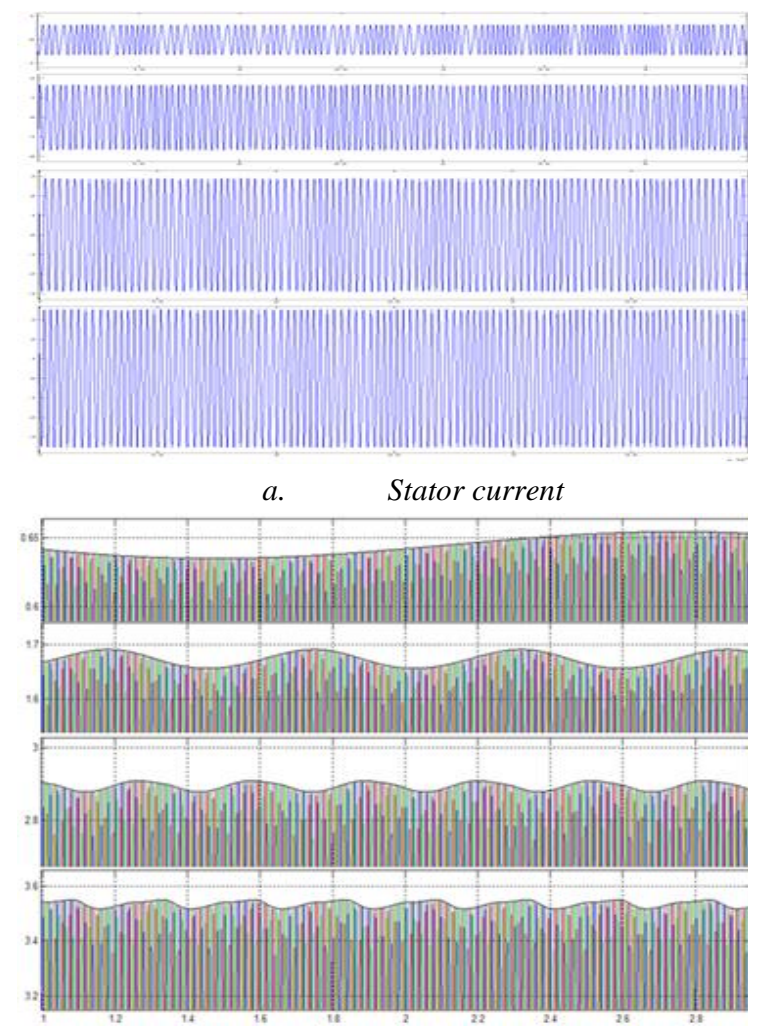

b. $\quad$ Stator current boundary appearance

Fig 5. Stator currents for two broken bars motor under $0,40 \%, 80 \%$ and $100 \%$ load.

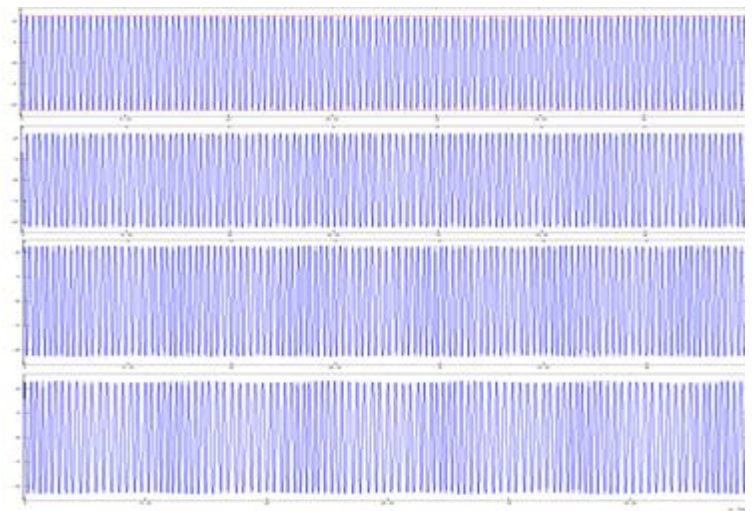

a. $\quad$ Stator current
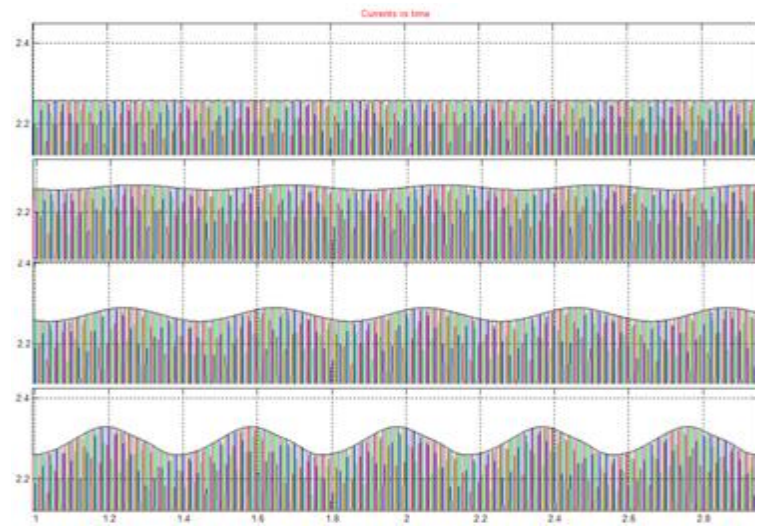

b. Stator current boundary appearance

Fig6. Stator currents for no, one, two, three broken bars under $60 \%$ load.
Motor load level changes and rotor fault severity have a direct impact to stator current amplitude as shown in Figure 4, Figure 5 and Figure 6 . They hardly affect the current waveform period. In contrast to that, rotor broken bars faults have an obvious influence to stator current period and the amplitude effect can be ignored as it too small.

For the purpose of quantities analysis, mean, absolute mean, standard deviation, mean absolute deviation, median and median absolute deviation are used as feature to represent broken rotor bar faults. Table 2 lists statistics values of stator current which can represent features of broken bar faults signals as shown in Figure 4, Figure5 and Figure6.

\subsection{WNN Based Feature Extraction}

Wavelet transform has been demonstrated as an effective approach in feature extraction in Table 2. However, for online broken rotor bars detection and diagnosis schemes, this method takes a considerable time in calculation, leading to delay whole diagnosis process. An accurate and on-line feature extraction approach is desired in industrial. WNN is introduced as a reliable solution method merging wavelet and neural net work together.

As shown in Figure 2, sampled stator current signal treated as input. These signals through wavelet layer and output layer to achieve expected targets. Figure 7(a) shows the output errors under BP training algorithm and proposed PSO algorithm. BP based network training algorithm has bigger error outputs comparing with PSO approach. Figure 7(b) illustrates the PSO based WNN training performance. The target mean squared error goal was reached after 398 epochs.

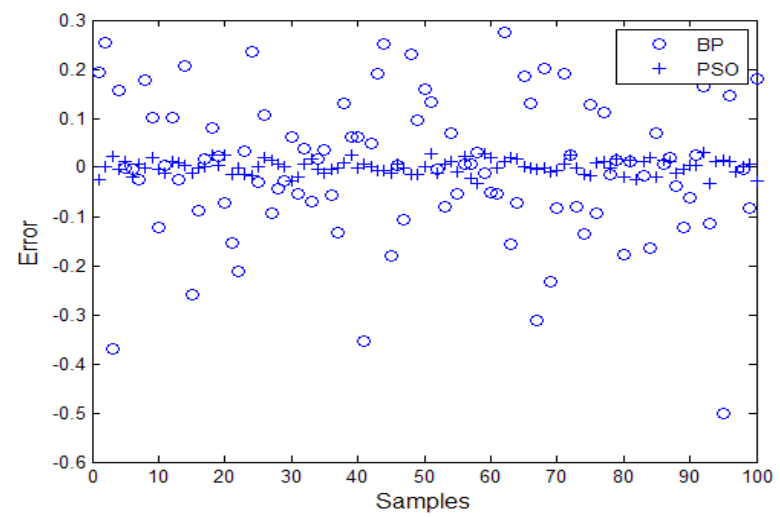

a. $\quad$ Output error of BP and PSO

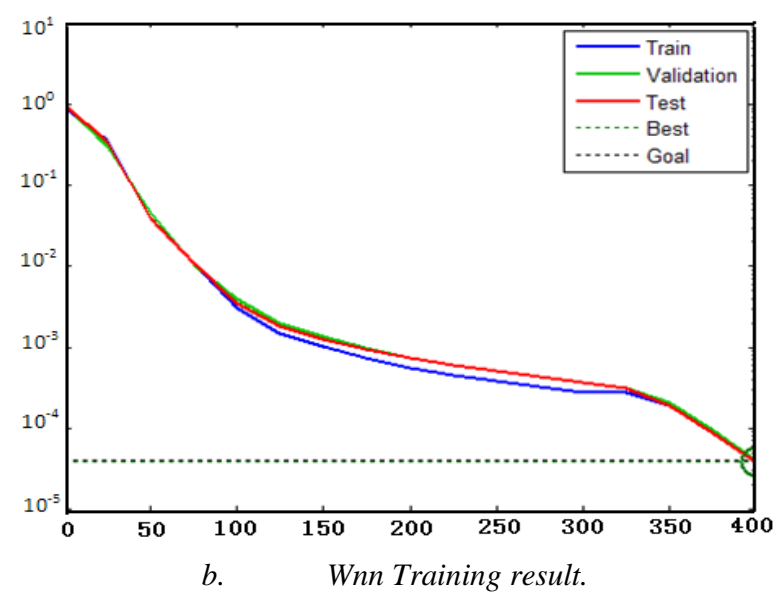

Fig 7. WNN Train performance 

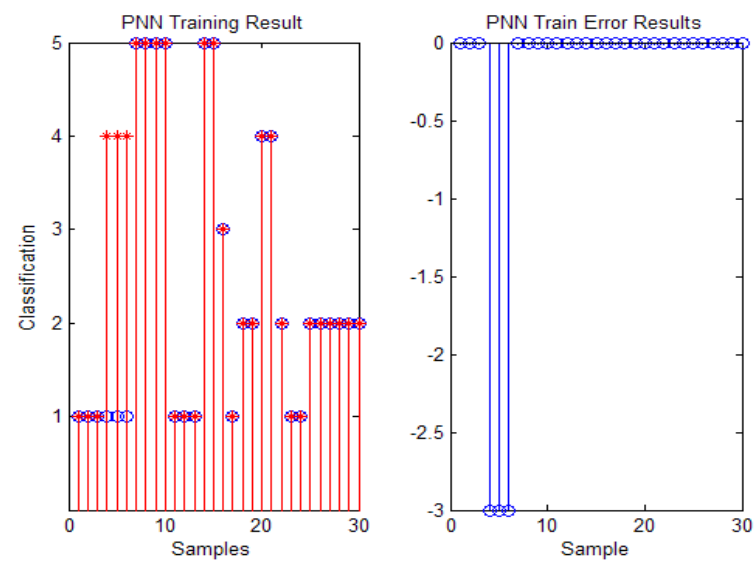

Fig 8. PNN training results

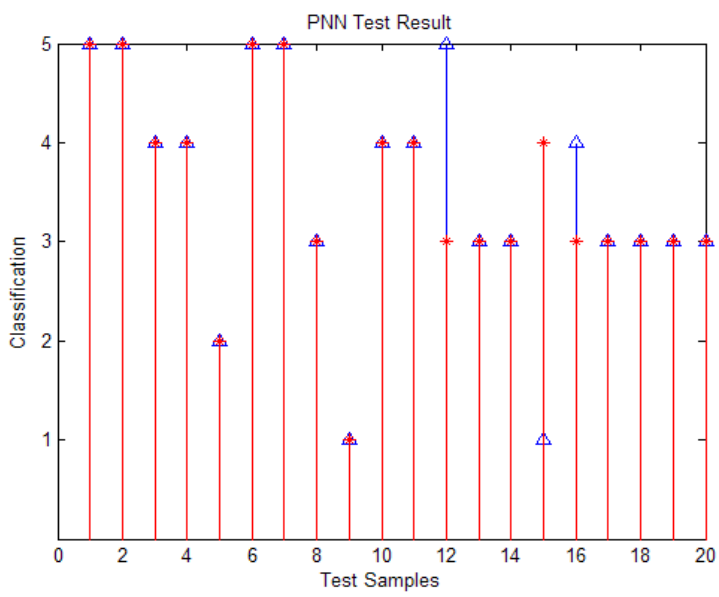

Fig 9. PNN training results

Table 2. Statistics values of stator current under $60 \%$ load.

\begin{tabular}{|c|c|c|c|c|c|c|}
\hline & mean & $\begin{array}{c}\text { abs } \\
\text { mean }\end{array}$ & std & $\begin{array}{c}\text { mean } \\
\text { abs } \\
\text { dev }\end{array}$ & median & $\begin{array}{c}\text { Median } \\
\text { abs. } \\
\text { dev }\end{array}$ \\
\hline Healthy & 0.0051 & 1.4576 & 1.605 & 1.449 & 0.0114 & 1.614 \\
\hline $\begin{array}{l}\text { One } \\
\text { Bars }\end{array}$ & $\begin{array}{c}- \\
0.0055 \\
\end{array}$ & 1.4603 & 1.6 & 1.44 & -0.01 & 1.602 \\
\hline $\begin{array}{l}\text { Two } \\
\text { bars } \\
\end{array}$ & $\begin{array}{c}- \\
0.0015 \\
\end{array}$ & 1.468 & 1.607 & 1.447 & -0.0089 & 1.607 \\
\hline $\begin{array}{l}\text { Three } \\
\text { bars }\end{array}$ & 0.0023 & 1.4829 & 1.615 & 1.451 & 0.0143 & 1.609 \\
\hline
\end{tabular}

Table 3. Test results of PNN

\begin{tabular}{|l|l|}
\hline $\begin{array}{l}\text { Number of training } \\
\text { examples }\end{array}$ & 30 \\
\hline $\begin{array}{l}\text { Number of testing } \\
\text { examples }\end{array}$ & 20 \\
\hline Training time(sec.) & 15 \\
\hline Testing time (sec) & 8 \\
\hline Accuracy rate & $85 \%$ \\
\hline
\end{tabular}

Table 4. Represent of output

\begin{tabular}{|c|c|}
\hline PNN output & $\begin{array}{c}\text { No of broken } \\
\text { bars }\end{array}$ \\
\hline 1 & Healthy \\
\hline 2 & 1 broken bar \\
\hline 3 & 2 broken bars \\
\hline 4 & 3 broken bars \\
\hline 5 & Other Faults \\
\hline
\end{tabular}

\subsection{Fault Classification}

To verify the feasibility of the proposed approach, PNN was used to classify the broken rotor bar faults. Six features generated from transient current signal were used to represent these faults.

First 30 samples were employed to train the PNN model as shown in Figure 8. Then 20 samples were used to test the proposed approach shown in Figure 9. The experimental results are shown in Table 3.

Figure 8 shows after 30 samples training, 3 samples out of 30 were misclassified. Red line represent target classification, blue line represents PNN classified results. Table 4 shows the represent conditions of PNN output.

\section{CONCLUSION}

This paper discussed a prototype of wavelet and probabilistic based neural network classifier for recognizing rotor bar defects under various transient signals. The proposed approach can reduce the quantity of extracted features of transient signals without losing its property. Moreover, this approach can also reduce the feature calculate time as well as memory space without jeopardising it diagnosis accuracy. Experimental results demonstrated that the proposed method has the ability of recognizing and classifying different broken bar type fault efficiently.

\section{REFERENCES:}

[1] Nandi, S and Toliyat, H, Fault diagnosis of electrical machine-a review, IEEE IEMCD'99, International Electric Machines and Drives Conference, May 912,Washington, USA, 1999, 219-221.

[2] Kliman, G B, et al., Noninvasive detection of broken rotor bars in operating induction motors, IEEE Transactions on Energy Conversion, EC-3(4), 1988, 873879.

[3] Cameron, J R, Thomson, W T and Dow, A B, Vibration and current monitoring for detecting airgap eccentricity in large induction motors, IEEE Proceedings, 133(pt. B, 3), 1986, 155-163.

[4] Schoen, R, et al., An unsupervised, on-line system for induction motor fault detection using stator current monitoring, IEEE Transactions on Industry Applications, 31(6), 1995, 1280-1286.

[5] Cruz, S.M.C and Cardoso, A.J.M, Stator winding fault diagnosis in three-phase synchronous and asynchronous motors by the extened park's vector approach, Conference Record of the 2000 IEEE Industry Applications Conference, CD-Rom, Roma, Italy, October 2000, $7 \mathrm{pp}$. 
[6] Nejjari, $\mathrm{H}$ and Benbouzid, $\mathrm{M}$, Monitoring and diagnosis of induction motors electrical faults using a current park's vector pattern learning approach, IEEE Transaction on Industry Applications, 36(3), 2000, 730-735.

[7] Salles, G, et al., Monitoring of induction motor load by neural network techniques, IEEE Transactions on Power Electronics, 15(4), 2000, 762-768.

[8] Chen, D S and Jain, R C, "A robust back propagation learning algorithm for function approximation," IEEE Trans. Neural Networks, vol. 5, no. 3, pp. 467-479, 1994.

[9] Zhang, Q and Benveniste, A, "Wavelet networks," IEEE Trans. Neural Networks, vol. 3, pp. 889-898, 1992.

[10] Pati, Y C and Krishnaprasad, P S, "Analysis and synthesis of feedforward neural networks using affine wavelet," IEEE Trans. Neural Neworks, vol. 4, no. 1, pp. $73-75,1993$.

[11] Zhang, J, et al., "Wavelet neural networks for function learning," IEEE Trans. Signal Process, vol. 43, pp. 14851497, 1995.

[12] Delyon, B, Juditsky, A and Benveniste, A, "Accuracy analysis for wavelet approximations," IEEE Trans. Neural Networks, vol. 6, pp. 332-348, 1995.

[13] Daubechies, I, "The wavelet transform, time-frequency localization, and signal anaylsis," IEEE Trans. Inform Theory, vol. 36, pp. 961-1005, 1990.

[14] Guo, Q J, Yu, H B and Xu, A D, "A Hybrid PSO-GD Based Intelligent Method for Machine Diagnosis," Digital Signal Processing, vol. 16, pp. 402-418, 2006.

[15] Kennedy, J and Eberhart, R C, "Particle swarm optimization," in: Proceedings of IEEE International Conference on Neural Networks, pp. 1942-1948, 1995.

[16] Kennedy, J, Eberhart, R C and Shi, Y, "Swarm Intelligence," Morgan Kaufmann, 2001.

[17] Abido, M A, "Optimal design of power system stabilizers using particle swarm optimization," IEEE Trans. Energy Convers, vol. 17, no. 3, pp. 406-413, 2002.

[18] Parsopoulos, K E and Vrahatis, M N, "Recent approaches to global optimization problems through particle swarm optimization," Natural Comput, vol. 1, pp. 235-306, 2002.

[19] Specht, D F, "Probabilistic neural networks," Neural Networks, vol. 3, no. 1, pp. 109-118, 1990.

[20] Masters, T, "Advanced algorithms for neural networks," a C++ sourcebook, New York: Wiley, 1995.

[21] Parzen, E, "On estimation of a probability density function and mode," The Annals of Mathematical Statistics, vol. 33, no. 3, pp. 1065-1076, 1962.

[22] Cacoullos, T, "Estimation of a multivariate density," Annals of the Institute of Statistical Mathematics, vol. 18, pp. 179-189, 1966

[23] Chow, M Y, Methodologies of using neural network and fuzzy logic technologies for motor incipient fault detection, s.l. : World Scientific Publishing Co.Pte.Ltd,Singapore, 1997.
[24] Patton, R J and Chen, J, "On-line residual compensation in robust fault diagnosis of dynamic systems," in IFAC Symp Artificial Intelligence in Real-time Control, Delft, The Netherlands, no. 17, pp. 221-227, 1992.

[25] Bertenshaw, D R, et al., "Detection of stator core faults in large electrical machines," IET Electric Power Applications, vol. 6, no. 6, pp. 295-301, 2012.

[26] Verma, S P and Natarajan, R, "Effects of eccentricity in induction motors," in Proc.Int.Conf.Electrical Machines,Budapest,Hungary, pp. 930-933, 1982.

[27] Walliser, R F and Landy, C F, "Determination of interbar current effects in the detection of broken rotor bars in squirrel cage induction motors," IEEE Trans.Energy Conversion, vol. 9, no. 1, pp. 152-158, 1994.

[28] Morita, I, "Air-gap flux analysis for cage rotor diagnosis," Elec.Eng.In Japan, vol. 112, no. 3, pp. 171$181,1992$.

[29] Ellison, A J and Yang, S J, "Effects of rotor eccentricity on acoustic noise from induction machines," Proc.Inst.Electr.Eng, vol. 118, no. 1, pp. 174-184, 1974.

[30] Toliyat, H A, Lipo, T A and White, J C, "Analysis of a concentrated winding induction machine for adjustable speed drive application part 1 (Motor analysis)," IEEE Transaction on Energy Conversion, vol. 6, no. 4, pp. 679-684, 1991

[31] Toliyat, H A and Lipo, T A, "Transsient analysis of cage induction machines under stator rotor bar and end-ring faults," IEEE Transaction on Energy Conversion, vol. 10, no. 2, pp. 241-247, 1995.

[32] Ah-jaco, A, "Modélisation des moteurs asynchrones triphasés en régime Transitoire avec saturation et harmoniques d'espace. Application au diagnostic," PhD thesis, Université de Lyon, juillet 1997.

[33] Munoz, A R and Lipo, T A, "Complex vector model of the squirrel-cage induction machine including instantaneous rotor bar currents," IEEE-IAP, vol. 35, no. 6,1999 .

[34] Lipo, T A and Toliyat, H A, "Feasibility study of a converter optimized induction motor," Palo Alto,CA,Electric Power Research Institute,EPRI Final Rep, pp. 2624-02, 1989.

[35] Luo, X, et al., "Multiple coupled circuit modeling of induction machines," IEEE Transaction on Industry apllications, vol. 31, no. 2, pp. 311-318, March/April 1995.

[36] AI-Nuaim, N A and Toliyat, H A, "A novel method for modeling dynamic air-gap eccentricity in synchronous machines based on modified winding function theory," IEEE Transaction on Energy Conversion, vol. 13, no. 2, pp. 156-162, June 1998.

[37] Houdouin, G, et al., "Coupled Magnetic Circuit Modeling of the Stator Windings Faults of Induction Machines Including Saturation Effect," In proceedings of the IEEE International Conference on industrial Technology(ICIT), pp. 148-153, 2004 